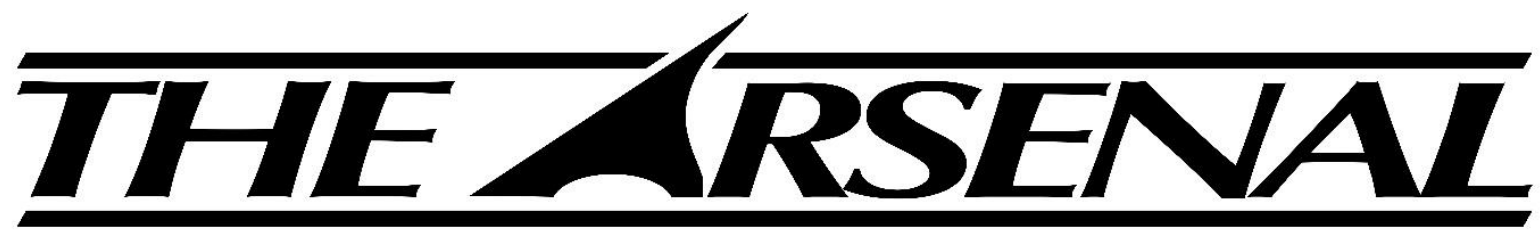

Augusta University's Undergraduate Research Journal

ISSN 2380-5064 | The Arsenal is published by the Augusta University Libraries | http://guides.augusta.edu/arsenal

Volume 3, Issue 2 (2020)

Special Edition Issue

FAST-TRACK EXTUBATION IN INFANCY AND EARLY CHILDHOOD FOLLOWING HEART SURGERY: OUTCOME ANALYSIS AND PREDICTORS OF FAILURE

Emma Geister, Raquel Esquivel, Danielle Crethers, Danalynn Weatherholt, Maria Gabriela Sanchez, Gustavo Munoz, and Anastasios C. Polimenakos

\title{
Citation
}

Geister, E., Esquivel, R., Crethers, D., Weatherholt, D., Sanchez, M. G., Munoz, G. $\&$ Polimenakos, A. C. (2020). Fast-track extubation in infancy and early childhood following heart surgery: Outcome analysis and predictors of failure. The Arsenal: The Undergraduate Research Journal of Augusta University, 3(2), http://doi.org/10.21633/issn.2380.5064/s.2020.03.02.12

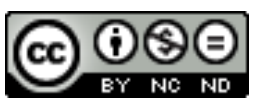

(C) Geister et al. 2020. This open access article is distributed under a Creative Commons Attribution NonCommercial-NoDerivs 2.0 Generic License (https://creativecommons.org/licenses/by-nc-nd/2.0/). 


\section{Fast-Track Extubation in Infancy and Early Childhood Following Heart Surgery: Outcome Analysis and Predictors of Failure}

Presenter: Emma Geister (Poster Presentation)

Authors: Emma Geister ${ }^{1}$, Raquel Esquivel ${ }^{1}$, Danielle Crethers ${ }^{2}$, Danalynn Weatherholt ${ }^{2}$, Maria Gabriela Sanchez ${ }^{2}$, Gustavo Munoz ${ }^{2}$, Anastasios C. Polimenakos²

Faculty Sponsor(s): Anastasios C. Polimenakos, MD

Department Affiliation: ${ }^{1}$ Biological Sciences, ${ }^{2}$ Medical College of Georgia

Funding: Augusta University CURS Summer Scholars Program

\section{ABSTRACT}

Early extubation (EE) has become a critical determinant in perioperative management following congenital heart surgery (CHS) during early childhood. Fast track (FT) strategies and EE, when feasible, can have beneficial effects on clinical outcomes. The authors sought to determine the impact of EE on clinical outcomes, total hospital costs, identify predictors of failure and suggested criteria for new patients. A retrospective chart review of children $\leq 6$ years old $(n=64)$ who underwent CHS between January-December 2017 was performed. EE was defined as successful removal of the endotracheal tube in the operating room or upon arrival in intensive care unit (ICU). Groups were identified as (A):EE/Fast track and (B):no EE. Determinants for EE failure were assessed, and cost analysis pursued. The authors found 39 patients with EE compared to 25 that were not. Children who were $E E$ (mean $=6.795$ days, $s d=4.250)$ spend significantly less $(\mathrm{p}<0.0001)$ overall time in the ICU compared to non-EE patients (mean=19.960 days, $s d=13.081$ ). The authors also found that the total hospital stay for patients who were EE (mean=6.976 days, $\mathrm{sd}=4.090$ ) was significantly reduced compared to those who were not (mean $=21.783$ days, $\mathrm{sd}=13.450)(\mathrm{p}<0.0001)$. Furthermore, the authors found that children who were EE had a significant reduction ( $\mathrm{p}<0.0001, \mathrm{sd}=23,196.203$ ) in total hospital cost than patients who were not EE. Based on our analysis, we concluded that EE is feasible following CHS during early childhood but requires team approach and thoughtful use of FT protocols.

Correspondence: Emma Geister, Augusta University, 1120 15 ${ }^{\text {th }}$ St. Augusta, GA 30912, egeister@augusta.edu 